\title{
Análise da Eficácia, Eficiência e Valor Acrescentado de Políticas Públicas Place-based - uma aplicação a territórios rurais
}

\author{
Anabela Santos ${ }^{1}$, Maria Manuel Serrano ${ }^{2}$ e Paulo Neto ${ }^{3}$
}

Resumo: O lançamento do Programa LEADER, em 1991, imprimiu uma nova dinâmica à política de desenvolvimento rural da União Europeia. Este programa, concebido para fomentar o empreendedorismo, potenciar o crescimento económico e estimular a inovação nas zonas rurais, distinguiu-se dos modelos clássicos de política pública por assentar numa abordagem territorial, multissectorial e integrada. Este artigo tem como principal objetivo a análise do valor acrescentado, da eficácia e da eficiência do Programa LEADER, na região Alentejo, ao longo das três primeiras fases da sua implementação. Para atingir este objetivo analisou-se a totalidade dos 2.706 projetos de investimento executados e financiados pelo LEADER, no período de 1991 a 2006, no Alentejo ${ }^{4}$, tendo sido consideradas no estudo as seguintes dimensões de análise: localização geográfica, tipo de promotor, atividade económica e natureza da despesa efetuada. A recolha de dados estatísticos, junto das entidades nacionais gestoras do Programa, permitiu a realização de uma análise estatística descritiva de indicadores financeiros e de impacto. Os resultados obtidos permitiram concluir que o LEADER contribuiu para uma nova dinâmica socioeconómica, uma vez que contribuiu para uma especialização do investimento realizado em torno de dois setores de atividade considerados hoje estratégicos para o Alentejo: o turismo e a agroindústria. Contudo, o LEADER revelou-se pouco expressivo nas despesas com I\&DT, precisamente aquelas atividades cujo contributo poderia alavancar a competitividade das empresas locais.

Palavras-chaves: Eficácia, eficiência, valor acrescentado, políticas públicas place-based, programa LEADER.

http://dx.doi.org/10.1590/1234-56781806-94790053s01003

1. Investigadora e doutoranda, iCite, Solvay Brussels School of Economics and Management, Université Libre de Bruxelles, Bélgica. E-mail: anabela.santos.mail@gmail.com

2. Professora Auxiliar, Universidade de Évora, Departamento de Sociologia e Socius-Iseg/UL, Coordenadora Setorial da UMPP-Unidade de Monitorização de Políticas Públicas da Universidade de Évora. E-mail: mariaserrano@uevora.pt

3. Professor Auxiliar com Agregação, Universidade de Évora, Departamento de Economia, Cefage-UÉ e Cieo-UAlg, Coordenador Geral da UMPP-Unidade de Monitorização de Políticas Públicas da Universidade de Évora. E-mail: neto@uevora.pt

4. Expressamos o nosso sincero agradecimento ao Sr. Rui Veríssimo Batista, Chefe do Projeto PIC Leader +, bem como aos Gabinetes de Ação Local do Alentejo e à Direcção-Geral de Agricultura e Desenvolvimento Rural, pela informação disponibilizada. 
Abstract: The launch of the LEADER program, in 1991, brought a new dynamic to the rural development policy of the European Union. This instrument designed to promote entrepreneurship, enhancing economic growth and stimulating innovation in rural areas, was distinct from the classical policy models because it is based on a territorial, multi-sectoral and integrated approach. This article aims to analyze the added value, the effectiveness and efficiency of the LEADER program in the Alentejo region, over the first three phases of its implementation. To achieve this goal we analyzed all of the 2.706 investment projects implemented and funded by LEADER, from 1991 to 2006, in Alentejo, under the following analytical dimensions: geographic location, type of promoter, economic activity and nature of expenditure made. The statistical data collected from the national program management entities allowed a descriptive statistical analysis of financial and impact indicators. The results showed that the LEADER program contributed to a new socio-economic dynamics in Alentejo, since it contributed to a specialization of investment around two economic sectors, now considered strategic for this region: tourism and agribusiness. Nevertheless the LEADER impact seems to had little significance regarding RED expenditure, precisely those activities whose contribution could leverage the competitiveness of local companies.

Key-words: Effectiveness, efficiency, added value, place-based public policies, LEADER program.

Classificação JEL: Z18, R58, R11.

\section{Introdução}

As políticas públicas place-based, focadas nas prioridades e potencialidades de um território ou região, têm assumido progressivamente um papel crescente, enquanto instrumento de desenvolvimento local. Um dos exemplos mais conhecidos de políticas placed-based aplicadas a territórios rurais é o Programa de Iniciativa Comunitária LEADER - Ligação entre Ações de Desenvolvimento da Economia Rural (OECD, 2006, p. 94). Segundo o Comité Económico e Social Europeu (CESE, 2011), ao longo dos últimos 20 anos, a abordagem LEADER demonstrou ser viável, o que levou este organismo a recomendar o alargamento da metodologia LEADER a outros programas operacionais para o período pós 2013. Inclusivamente, o Quadro Estratégico Comum (QEC), delineado pela Comissão para o período 2014-2020, pressupõe como condição prévia de acesso ao Fundo Europeu de Desenvolvimento Regional (FEDER), a existência de Estratégias de Investigação e Inovação Nacionais/Regionais para a Especialização Inteligente (RIS3), em cada Estado-membro (FORAY et al., 2012, p. 12).

O Programa de Iniciativa Comunitária LEADER surge em 1991, num contexto marcado por profundas mutações da estrutura econó- mico-social da sociedade rural, nomeadamente: i) propensão para o envelhecimento populacional; ii) fraca concentração demográfica e iii) reduzido rendimento per capita. Face à incapacidade das teorias sectoriais e/ou exógenas para alterar a tendência de declínio do mundo rural, a Comissão Europeia veio defender, no documento "O Futuro do Mundo Rural", publicado em 1988, a necessidade de ser experimentada uma nova abordagem para o desenvolvimento rural, a qual deveria ter um enfoque territorial e uma intervenção das comunidades locais na procura das soluções (CHAMPETIER, 2003). Paralelamente, assiste-se, na mesma data, a uma reformulação do papel dos fundos estruturais europeus com o Regulamento CE no 2052/88, o qual veio prever a necessidade de uma "maior concentração dos instrumentos públicos nas regiões mais carenciadas, [...] [tornando] quase inevitável a criação de um programa inovador para contrariar o despovoamento e o ciclo de empobrecimento das áreas rurais" (MORENO, 2003, p. 3). É precisamente neste contexto que foi criado o Programa LEADER, o qual foi usado para canalizar recursos financeiros para zonas mais desfavorecidas, como os territórios rurais de baixa densidade populacional, com dificuldades de acesso ao financiamento externo (OECD, 2006). 
Os sistemas de incentivos ao investimento são instrumentos de política pública que têm por objetivo estimular o empreendedorismo, dinamizar a economia e fomentar a coesão territorial. Segundo Marques e Santos (2011), os estímulos ao investimento podem alavancar o desenvolvimento regional e as políticas públicas descentralizadas podem motivar trajetórias de especialização produtiva. Contudo, Dall'erba et al. (2008) já tinham questionado a eficácia das políticas estruturais quanto à promoção da convergência regional e à sustentação do crescimento económico nas regiões mais desfavorecidas.

O presente estudo pretende contribuir para o debate público sobre a eficácia, a eficiência e o valor acrescentado de políticas públicas place-based, vocacionadas para territórios rurais ${ }^{5}$, mediante a análise das características do investimento realizado na região Alentejo, no âmbito do Programa LEADER, entre 1991 e 2006.

O Alentejo ${ }^{6}$, região com forte tradição agrícola, estende-se por cerca de $27.300 \mathrm{~km}^{2}$ e representa cerca de 1/3 do território de Portugal continental. Os indicadores demográficos deste território, em 2011, revelavam uma densidade populacional de $18,6 \mathrm{hab} / \mathrm{km}^{2}$, comparativamente a uma média nacional de $114,3 \mathrm{hab} / \mathrm{km}^{2}$, e um decréscimo da população residente, face ao período de 1970, em cerca de 14\% (INE, 2012; SANTOS, 2012, p. 61). Por outro lado, nas últimas décadas, "o Alentejo não [melhorou] a sua posição no que respeita a indicadores de competitividade territorial e [...] não [tem] vindo a verificar sinais [significativos] de convergência para a média nacional e da União Europeia" (Universidade de Évora, 2007, p. 2).

A investigação que suporta este artigo, procurou responder às seguintes questões: Quais os

5. No âmbito do Programa LEADER, entende-se por zonas rurais os territórios do interior ou litoral com uma densidade populacional igual ou inferior a 150 habitantes por $\mathrm{km}^{2}$, onde a maior parte das terras são utilizadas para a agricultura, por atividades económicas e culturais próprias aos habitantes desta região (BARTHELEMY e VIDAL, 1995).

6. Para efeitos de delimitação geográfica do Alentejo consideraram-se as sub-regióes Alto Alentejo, Alentejo Central, Alentejo Litoral e Baixo Alentejo. Não foi considerada a atual NUTS III Lezíria e Vale do Tejo. principais beneficiários do programa? Que tipo de atividades e de despesas foram financiadas? Como influenciou o programa os investimentos em regiões menos povoadas, mais envelhecidas ou com fraca concentração empresarial? Os efeitos alcançados corresponderam aos objetivos pretendidos? Os recursos canalizados conduziram aos resultados esperados?

Com o propósito de responder às questões levantadas, estruturou-se o artigo nos seguintes pontos: i) Fundamentação Teórica - aborda a temática das políticas públicas place-based e identifica as dimensões de análise destas políticas; ii) Metodologia - procede à descrição da metodologia e dos procedimentos metodológicos utilizados no processo de investigação; iii) Resultados e Discussão - apresenta e analisa os resultados obtidos e iv) Conclusões - sintetiza as conclusões obtidas acerca da eficácia, da eficiência e do valor acrescentado do Programa LEADER na região Alentejo.

\section{Fundamentação teórica}

\subsection{Políticas públicas place-based - o caso do Programa LEADER}

As políticas públicas place-based assentam num modelo de governação territorial, onde o poder de decisão se encontra descentralizado nos atores locais e o plano de ação é delineado com base nas potencialidades e necessidades de uma identidade geográfica definida (REIMER e MARKEY, 2008). Esta descentralização pode reforçar a eficácia e a eficiência do sector público, comparativamente às políticas públicas definidas ao nível nacional (BERTHET, 2008, p. 134). Contudo, um dos desafios com que se depara a execução das políticas territoriais é a dificuldade de serem definidas unidades territoriais uniformes e mobilizar diversos atores em torno de um projeto comum, sobretudo, numa sociedade marcada essencialmente pelo individualismo (LAZAREV, 2009, p. 204). Segundo Barca et al. (2012, p. 148), para que a implementação de políticas place-based seja 
bem-sucedida é necessário assegurar que os estímulos aos comportamentos de todos os parceiros estejam alinhados em torno do mesmo objetivo. Neste contexto, apenas com uma forte articulação entre os conceitos place-based e people-based é que se conseguem implementar políticas que conduzam ao desenvolvimento regional (BARCA et al., 2012, p. 149).

A abordagem place-based constitui a espinha dorsal da política de coesão para o período de 20142020, inclusivamente a existência de "Estratégias de Investigação e Inovação Nacionais/Regionais para a Especialização Inteligente" (RIS3) em que cada Estado-membro constitui um requisito $e x$ -ante de acesso aos fundos estruturais. As estratégias RIS3 defendem que os territórios deverão dar prioridade aos recursos endógenos, aos setores de atividade e/ou tecnologias, nos quais possuem uma vantagem comparativa e potencial para alavancarem atividades inovadoras (FORAY et al., 2012 , p. 9). Esta iniciativa foi prevista para potenciar a inovação no sentido lado, ou seja, não apenas no domínio da investigação, mas também no fomento a novos modelos de negócios ou organizacionais (FORAY et al., 2012, p. 10). O conceito RIS3 foi desenvolvido com base nas aprendizagens oriundas de anteriores Estratégias Regionais de Inovação Europeias, cujas avaliações evidenciaram algumas limitações em termos de resultados e de eficácia (FORAY et al., 2012, p. 11-12).

Com este estudo procurou-se dar continuidade às investigações realizadas sobre esta temática, mediante a análise da eficiência, da eficácia e do valor acrescentado de um dos exemplos mais conhecido de políticas place-based: o Programa de Iniciativa Comunitária LEADER. Quando surge em 1991, o Programa LEADER vem defender princípios distintos dos sustentados pelas teorias clássicas: uma abordagem bottom-up, em vez da abordagem tradicional top-down e um enfoque territorial, em oposição ao enfoque sectorial, conferindo-lhe a capacidade de abordar a problemática do desenvolvimento rural com base num método inovador (SANTOS, 2012, p. 21).

Nessa época, preconizava-se que a criação de condições para o aparecimento de novas ativida- des complementares (ou não) ao setor agrícola serviria de alavanca para o aparecimento de oportunidades de emprego alternativas e de outras fontes de rendimentos, com vista a melhorar a qualidade de vida no meio rural (CHAMPETIER, 2003). Para a concretização destes objetivos, o sector privado, o sector público e o terceiro sector, foram convidados a participar na elaboração dos programas locais (LAZAREV, 2009, p. 191). Estas parcerias deram origem à constituição dos Grupos de Ação Local (GAL), entidades responsáveis pela definição, organização e implementação da Estratégia Local de Desenvolvimento (ELD) e pela seleção dos projetos a serem financiados (CE, 2006, p. 10-15). A diversidade das zonas rurais e a autonomia dos GAL, na transposição dos objetivos globais ao nível local, originou o aparecimento de ELD na mesma proporção e número que estas entidades. Na União Europeia, ao longo das três primeiras iniciativas do Programa LEADER, que decorreram de 1991 a $2006^{7}$, a sua área de intervenção passou de $367.000 \mathrm{Km}^{2}$ para $1.577 .386 \mathrm{Km}^{2}$ e o número de GAL a atuar nessas zonas aumentou de 217 para 893 (CE, 2006, p. 7). Em Portugal, o LEADER I deu origem à constituição de $20 \mathrm{GAL}$, dois dos quais localizados na região Alentejo. Com o LEADER + , Portugal passou a contar com a presença de 52 GAL e o Alentejo com 8 entidades (BARROCAS, 2008), conforme ilustra a Figura 1.

Apesar dos GAL terem autonomia para definirem as orientações da EDL, em função das prioridades e potencialidades que identificassem para o território, os resultados finais teriam de convergir para os objetivos gerais definidos a nível comunitário para o Programa LEADER, no Parecer CE no 91/C (CES, 1991), nomeadamente: i) atenuar a desertificação humana e o envelhecimento populacional; ii) diversificar o tecido empresarial; iii) valorizar e promover os recursos endógenos do território; iv) estimular iniciativas e projetos inovadores; v) desenvolver competências, mediante a formação e qualificação dos

7. LEADER I de 1991 a 1993, LEADER II de 1994 a 1999 e LEADER + de 2000 a 2006. 
Figura 1. Três iniciativas LEADER na região Alentejo - mapa com a evolução das zonas de intervenção dos GAL

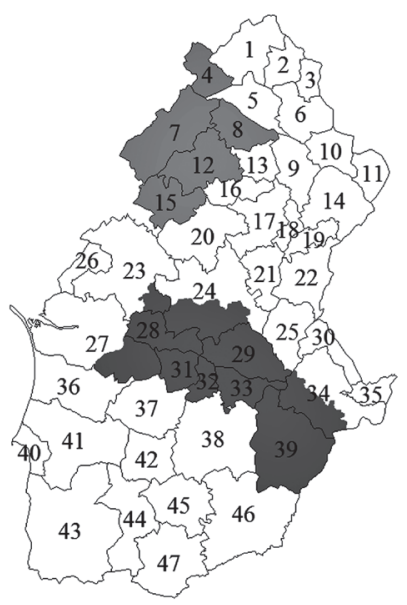

LEADER I

(1991 - 1993)

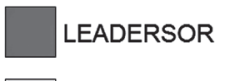

ALENTEJO XXI

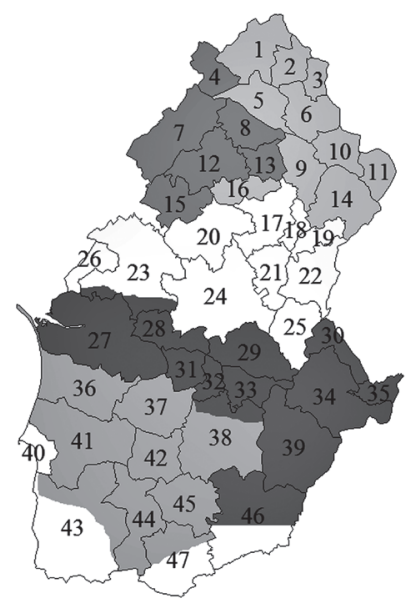

LEADER II

(1994 - 1999)

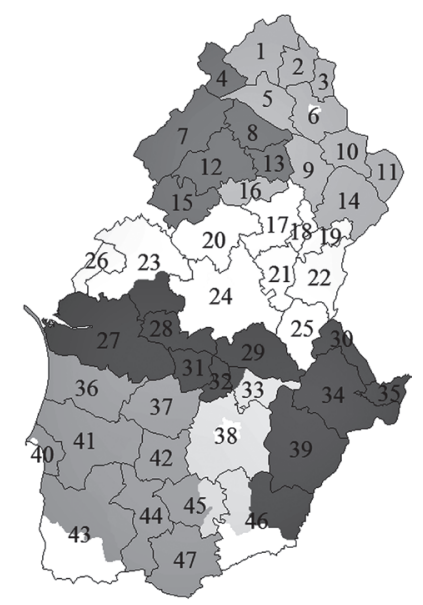

LEADER +

(2000 - 2006)

TERRAS DENTRO

ADL

\begin{abstract}
1. Nisa / 2. Castelo de vide / 3. Marvão / 4. Gavião / 5. Crato / 6. Portalegre / 7. Ponte de Sôr / 8. Alter do Chão / 9. Monforte / 10. Arronches / 11. Campo Maior/12. Avis / 13. Fronteira/14. Elvas / 15. Mora / 16. Sousel/ 17. Estremoz/18. Borba / 19. Vila Viçosa / 20. Arraiolos / 21. Redondo / 22. Alandroal / 23. Montemor-o-Novo / 24. Évora / 25. Reguengos de Monsaraz / 26. Vendas Novas / 27. Alcácer do Sal / 28. Viana do Alentejo / 29. Portel/30. Mourão/31. Alvito / 32. Cuba/33. Vidigueira/ 34. Moura / 35. Barrancos / 36. Grândola/37. Ferreira do Alentejo/38. Beja / 39. Serpa / 40. Sines / 41. Santiago do Cacém/ 42. Aljustrel / 43. Odemira / 44. Ourique / 45. Castro Verde/46. Mértola/47. Almodôvar
\end{abstract}

Fonte: Neto, Santos e Serrano (2012, p. 638).

recursos humanos ou vi) fomentar a preservação do meio ambiente. A presente análise irá centrar-se precisamente sobre estas metas.

\subsection{Dimensões de análise de políticas públicas: eficácia, eficiência e valor acrescentado}

A análise das políticas públicas é geralmente baseada na apreciação de três elementos: os "recursos financeiros e não monetários" (input), canalizados para a implementação e execução de uma política ou programa, os quais se traduzem em "realizações materiais e imateriais" (output) e os "resultados ou efeitos" (outcome), gerados numa economia ou território (EC, 2008; VOLLET e HADJAB, 2008), conforme ilustra a Figura 2.
Neste contexto, "a análise da eficácia e eficiência está baseada na apreciação das relações existentes entre os inputs, outputs e outcomes" (MANDL et al., 2008, p. 2). A análise de eficiência verifica se os recursos mobilizados produziram os resultados, efeitos e impactos pretendidos e a análise de eficácia examina se os objetivos fixados foram alcançados (EC, 2008, p. 42). Quando a análise de eficiência é expressa em termos monetários, o rácio input-output é o indicador mais vulgarmente utilizado para medir a performance da política ou programa. A sua interpretação indica quanto foi despendido para se produzir um determinado output (SAPRU, 2011, p. 33). Este indicador corresponde também, em sentido lato, à "análise custo-eficácia", a qual tem por finalidade comparar as despesas de um programa com 
Figura 2. Dimensões da avaliação de políticas públicas

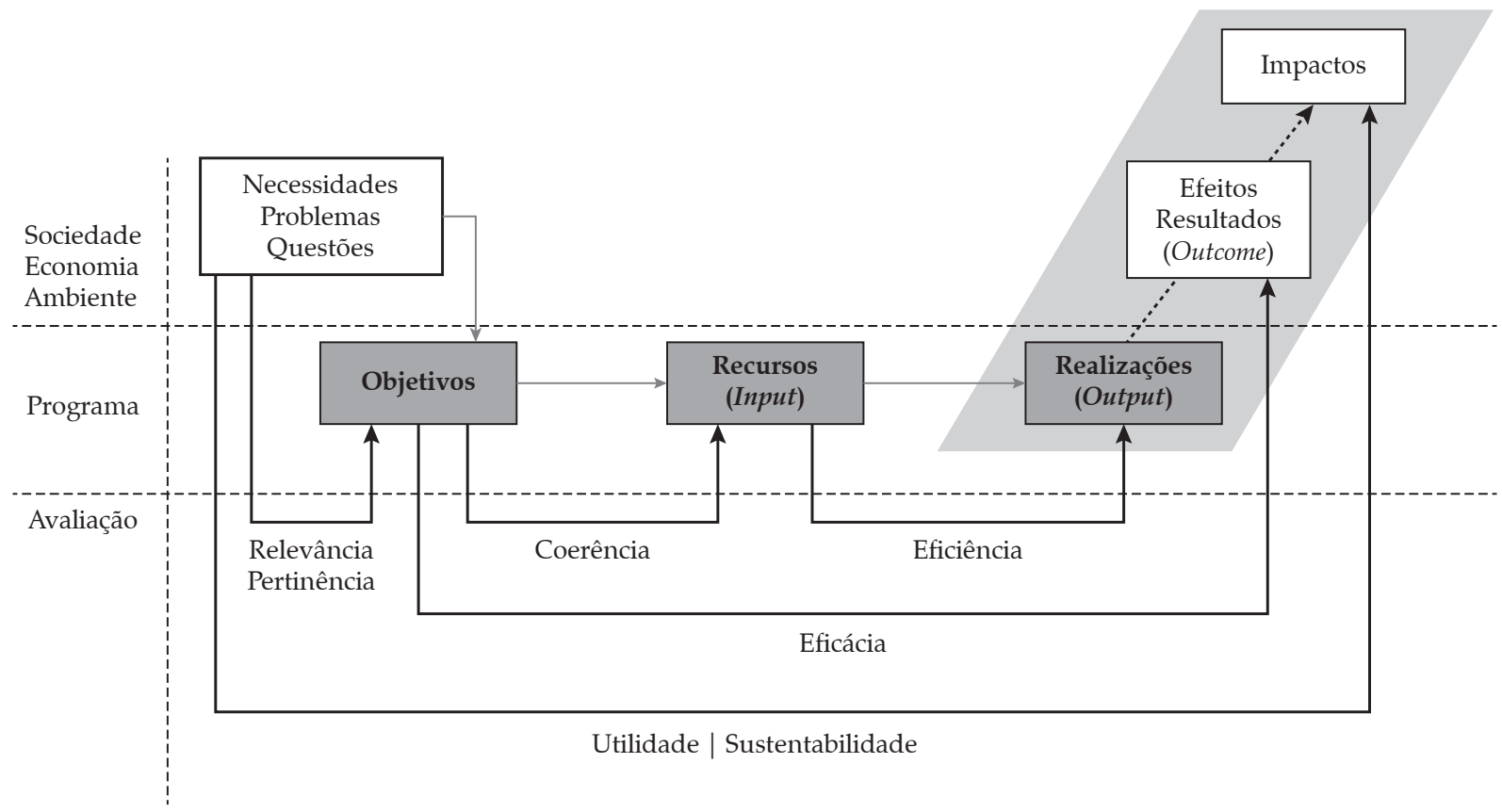

Fonte: Santos (2012, p. 35) adaptado com base em EC (2008, p. 42) e Arnaud e Boudeville (2004, p. 37).

os resultados obtidos, para determinar, por exemplo, o custo por emprego criado (EURÉVAL, 2010, p. 1).

No presente trabalho, foram considerados como inputs os recursos financeiros utilizados na implementação do LEADER e como outputs o investimento realizado ao abrigo deste Programa na região Alentejo, no período de 1991 a 2006. Os outcomes assumem uma dimensão mais qualitativa, baseada numa análise comparativa do diagnóstico ${ }^{8}$ da região Alentejo, antes e após a implementação das três fases do programa.

Os Fundos Comunitários são suscetíveis de gerar, quando utilizados de forma acertada, um valor acrescentado face a uma situação alternativa caracterizada pela ausência da intervenção

8. Por via da análise SWOT (Strengths, Weaknesses, Opportunities, and Threats) identificaram-se os pontos fortes, os pontos fracos, as oportunidades e as ameaças de uma entidade ou território; síntese realizada pelas entidades regionais gestoras dos fundos comunitários (CCRA, 1986; AUTORIDADE DE GESTÃO DO INALENTEJO, 2008). pública (CE, 2002, p. 3) $)^{9}$. As políticas ou programas geram outputs e outcomes, os quais, com base neste princípio, não existiriam, total ou parcialmente, sem os inputs que estas injetam numa determinada realidade económica e social. Segundo a EC (2008, p. 42), esta situação traduz-se na utilidade da política pública, a qual está associada à noção de valor acrescentado. A Figura 3 resume os quatro temas-chaves que agrupam alguns indicadores, quantitativos e qualitativos, que permitem a medição do valor acrescentado, definidos pela CE (2002).

9. Neste contexto é igualmente importante averiguar se o promotor poderia ter sido capaz de desenvolver o projeto mesmo sem beneficiar deste apoio (TCE, 2010, p. 30). Numa situação designada de "peso morto", a subvenção não produz nenhum efeito adicional, uma vez que o projeto subsidiado teria sido total ou parcialmente executado sem a concessão desta ajuda financeira. Um forte indício da existência de um "peso morto" é o facto de o promotor já ter iniciado o projeto antes de ter sido notificado da decisão de análise (TCE, 2010, p. 30). No caso concreto deste estudo, em virtude de não ser conhecido o calendário de execução de cada candidatura, partiu-se do princípio que os resultados gerados não teriam ocorrido sem a existência do programa. 
Figura 3. Indicadores de medição do Valor Acrescentado Comunitário

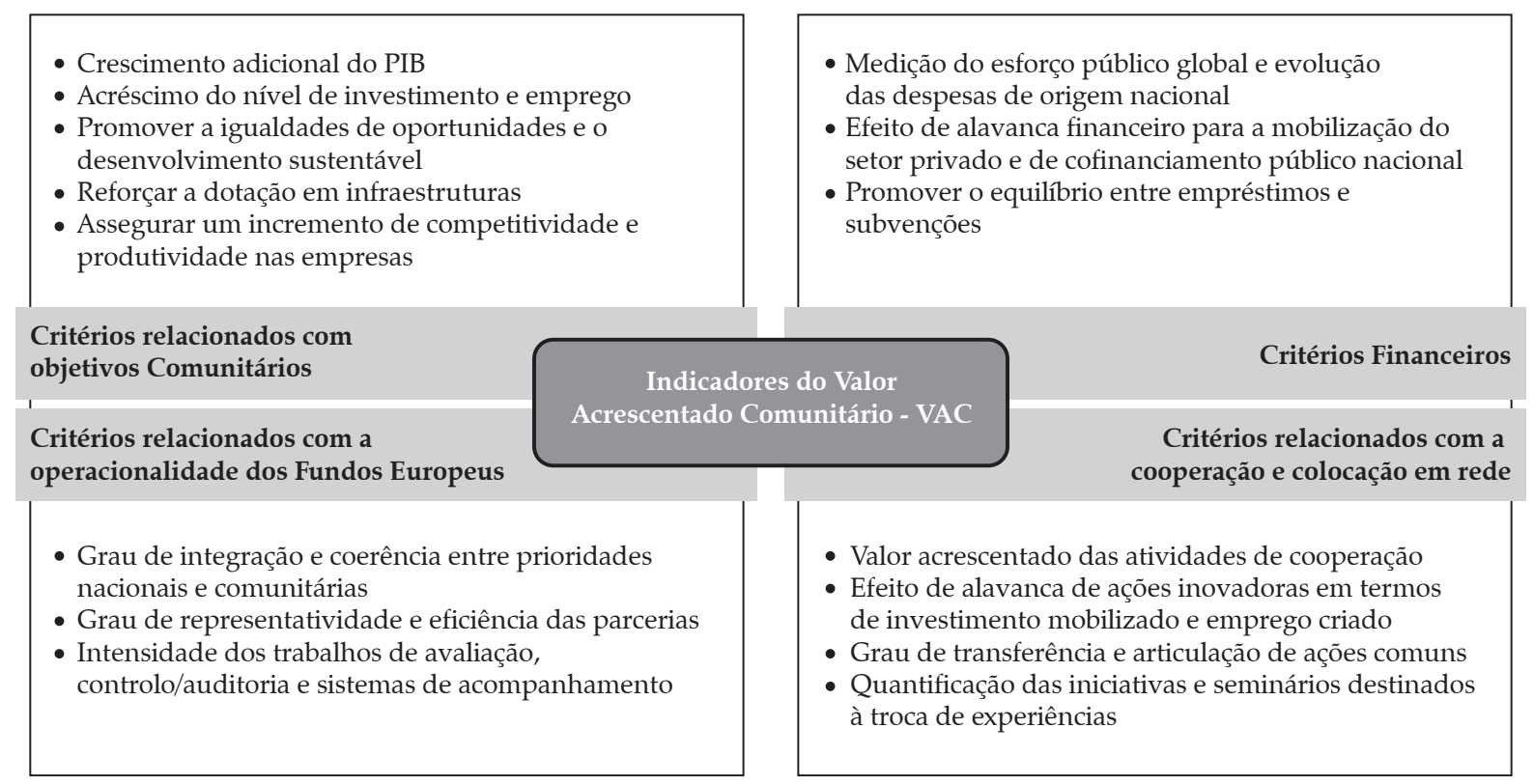

Fonte: Elaborado pelos autores com base em CE (2002).

Contudo, o valor acrescentado do Programa LEADER não se limita ao impacto produzido pelos incentivos financeiros introduzidos nas economias rurais. "A abordagem LEADER implica custos e riscos adicionais em relação a métodos mais tradicionais e centralizados de execução da política de desenvolvimento rural, mas tem igualmente potencial para gerar valor acrescen- tado [adicional] através das suas características metodológicas" (TCE, 2010, p. 12), conforme ilustra a Figura 4.

Segundo Wade e Rinne (2008, p. 82), o LEADER é um Programa cujos efeitos são mais visíveis no longo prazo, como resultado do desenvolvimento progressivo da capacidade e do envolvimento dos recursos humanos. Neste con-

Figura 4. Evolução da Eficiência dos Programas-tipo LEADER versus Programas clássicos

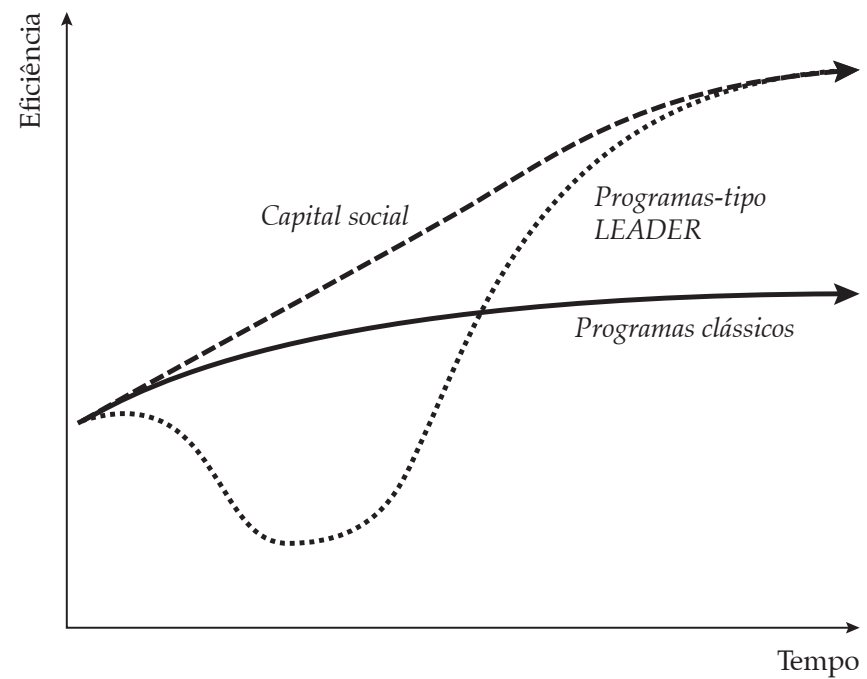

Fonte: Adaptado pelos autores com base em ÖIR (2004, p. 57). 
texto, quanto maior é o período de experiência e familiarização das regiões/países com o Programa LEADER, mais expressivos são os resultados observados (WADE e RINNE, 2008, p. 82).

\section{Metodologia}

Em Portugal, as avaliações realizadas ao Programa LEADER tiveram essencialmente por base a análise da taxa de execução financeira. Foi, aliás, com base neste indicador que os técnicos apreciaram a eficácia desta iniciativa comunitária (SANTOS, 2012, p. 42). Os indicadores de resultados destas análises incidiram principalmente no investimento realizado por domínios e tipo de promotor e os indicadores de impacto centraram-se no emprego criado e respetivo perfil (SANTOS, 2012, p. 42).

Neste estudo ${ }^{10}$ procurou-se efetuar uma análise mais aprofundada do investimento realizado ao abrigo deste Programa, com enfoque na região Alentejo. Contudo, nem toda a informação necessária para este trabalho de investigação estava disponível nos relatórios de execução e de avaliação final do Programa LEADER. Perante esta lacuna, foi necessário proceder à análise individual dos 2.706 projetos de investimento executados e financiados pelo Programa LEADER na região Alentejo, entre 1991 e 2006. Com base nesta apreciação foi elaborada uma base de dados onde cada projeto se encontra classificado por localização geográfica, tipo de promotor, atividade económica e natureza da despesa efetuada. O critério que presidiu à escolha das variáveis de análise baseou-se nos objetivos da iniciativa LEADER, com vista à caracterização das realizações e resultados desta iniciativa comunitária no Alentejo.

A análise dos resultados teve por base a apreciação de indicadores de realização e resultado e do rácio input-output, sendo este último obtido

10. O qual é o resultado de um trabalho de investigação mais extenso realizado no âmbito de uma dissertação de Mestrado em Economia na Universidade de Évora (SANTOS, 2012). pelo quociente entre os custos (input) e os resultados obtidos (output).

As entidades consultadas para a obtenção da informação foram os Grupos de Ação Local a atuarem na região Alentejo; a Comissão Gestora do LEADER+; a Direcção-Geral de Agricultura e Desenvolvimento Rural; a Comissão Europeia - Unidade F1. Programas de Desenvolvimento Rural (Bruxelas); a ELARD - European LEADER Association for Rural Development (Bruxelas) e a Federação Minha Terra.

\section{Resultados e discussão}

\subsection{Apresentação dos resultados globais}

Ao longo das três primeiras fases do Programa LEADER, que decorreram de 1991 a 2006, foram executados e financiados 2.706 projetos de investimento na região Alentejo, num valor total de 85,5 milhões de euros. A contribuição da CE foi equivalente a cerca de 47 milhões de euros e a despesa privada a 27,8 milhões de euros. No âmbito do emprego, previa-se a criação de 1.177 postos de trabalho e a manutenção de 1.152. Na realidade, em termos absolutos, o número de postos de trabalho criados aumentou ao longo das 3 fases do programa, contudo o esforço financeiro necessário para a criação de um posto de trabalho adicional também aumentou em 55\%, entre o LEADER II e o LEADER +. Do ponto de vista social e no domínio do mercado de trabalho, este facto pode ser sintomático de que o programa ganhou em eficácia - resultados versus objetivos - mas perdeu em eficiência - recursos versus objetivos entre a $2^{\mathrm{a}}$ e $3^{\mathrm{a}}$ fase do Programa (ver Quadro 1).

Uma conclusão similar à anterior (ganho em eficácia e perda de eficiência) pode ser sugerida pela análise da evolução do rácio input-output, respeitante ao acréscimo do nível de emprego entre o LEADER II e o LEADER + . Já no que respeita ao efeito de alavanca financeira na despesa privada, no cofinanciamento público nacional e no acréscimo do nível de investimento, verifica-se um aumento da eficiência do LEADER II para o LEADER + (ver Quadro 2). 
Quadro 1. Indicadores de Realização e de Resultado, LEADER I - LEADER + , região Alentejo

\begin{tabular}{|c|c|c|c|c|}
\hline & $\begin{array}{l}\text { LEADER I } \\
(1991-1994) \\
\end{array}$ & $\begin{array}{l}\text { LEADER II } \\
(1995-1999)\end{array}$ & $\begin{array}{l}\text { LEADER + } \\
(2000-2006) \\
\end{array}$ & Total \\
\hline \multicolumn{5}{|l|}{ Indicadores de Realização (Output) } \\
\hline № projetos aprovados e executados & 202 & 1.000 & 1.504 & 2.706 \\
\hline Investimento realizado & $11.970 .714 €$ & $25.875 .238 €$ & $47.583 .243 €$ & $85.429 .195 €$ \\
\hline \multicolumn{5}{|l|}{ Despesa pública } \\
\hline Contribuição CE & $6.078 .128 €$ & $15.865 .147 €$ & $25.222 .983 €$ & $47.166 .258 €$ \\
\hline Contribuição nacional & $412.583 €$ & $1.905 .235 €$ & $8.124 .127 €$ & $10.441 .945 €$ \\
\hline Despesa privada & $5.480 .003 €$ & $8.104 .856 €$ & $14.236 .133 €$ & $27.820 .992 €$ \\
\hline Postos de trabalho criados & 167 & 462 & 548 & 1.177 \\
\hline Emprego mantido & n.d.* & 203 & 949 & 1.152 \\
\hline \multicolumn{5}{|l|}{ Indicadores de Resultado (Outcome) } \\
\hline Emprego médio criado por projeto & 0,83 & 0,46 & 0,36 & 0,43 \\
\hline Investimento por posto de trabalho criado & $71.681 €$ & $56.007 €$ & $86.831 €$ & $72.582 €$ \\
\hline
\end{tabular}

* Não disponível.

Fonte: Cálculos elaborados pelos autores com base em Ministério da Agricultura (1995), Madrp (2001), Autoridade de gestão do Pic LEADER+ (2008) e Barrocas (2008)

Quadro 2. Rácio input-output, LEADER I - LEADER +, região Alentejo

\begin{tabular}{|c|c|c|c|}
\hline & LEADER I & LEADER II & LEADER + \\
\hline \multicolumn{4}{|c|}{ Objetivo: análise do efeito de alavanca financeiro na despesa privada* } \\
\hline Rácio Input-Output & $1,1 €$ & $2,0 €$ & $1,8 €$ \\
\hline \multicolumn{4}{|c|}{ Objetivo: análise do efeito de alavanca financeiro no cofinanciamento público nacional ${ }^{* *}$} \\
\hline Rácio Input-Output & $14,7 €$ & $8,3 €$ & $3,1 €$ \\
\hline \multicolumn{4}{|c|}{ Objetivo: análise do acréscimo do nível de investimento ${ }^{* * *}$} \\
\hline Rácio Input-Output & $0,51 €$ & $0,61 €$ & $0,53 €$ \\
\hline \multicolumn{4}{|c|}{ Objetivo: análise do acréscimo do nível de emprego ${ }^{* * * *}$} \\
\hline Rácio Input-Output & $36.396 €$ & $34.340 €$ & $46.027 €$ \\
\hline
\end{tabular}

${ }^{*}$ Input $=$ Contribuição CE $\mid$ Output $=$ Despesa privada.

** Input $=$ Contribuição CE $\mid$ Output $=$ Cofinanciamento público nacional.

*** Input $=$ Contribuição CE $\mid$ Output $=$ Investimento realizado.

**** Input $=$ Contribuição CE $\mid$ Output $=$ Emprego criado.

Fonte: Elaborado pelos autores com base no Quadro 1.

\subsection{Análise descritiva do investimento realizado}

O sector privado foi, nas três iniciativas LEADER, aquele que mais contribuiu para a Formação Bruta de Capital Fixo na região, por via do investimento realizado. Contudo, as entidades deste sector registaram uma diminuição do seu peso relativo, comparativamente ao $3^{\text {o }}$ setor e ao setor público. No mesmo período, os GAL foram responsáveis por $26 \%$ do investimento realizado, assumindo a importância da despesa realizada uma proporção tendencialmente constante (ver Quadro 3).
Em média, 96\% do investimento realizado pelo setor privado assentou em projetos exclusiva ou prioritariamente baseados na aquisição de equipamento e/ou de obras de remodelação. Os pedidos de apoio, predominantemente fundamentados em investimentos nas áreas do marketing e comunicação e da aquisição de conhecimentos, apresentam um peso relativo residual, entre $1 \%$ a $2 \%$ do investimento total. As principais atividades económicas financiadas foram as atividades turísticas (alojamento e animação) e a agro-indústria. O desenvolvimento de atividades no setor dos serviços apresentou uma tendência 
Quadro 3. Investimento realizado por tipologia de promotor, LEADER I - LEADER +, região Alentejo

\begin{tabular}{|l|c|c|c|c|}
\hline & LEADER I & LEADER II & LEADER + & Conjunto \\
\hline Setor Privado & $66 \%$ & $41 \%$ & $40 \%$ & $\mathbf{4 6 \%}$ \\
\hline Setor Público & $3 \%$ & $13 \%$ & $12 \%$ & $\mathbf{1 0 \%}$ \\
\hline 3o Setor & $8 \%$ & $17 \%$ & $23 \%$ & $\mathbf{1 8 \%}$ \\
\hline GAL & $23 \%$ & $29 \%$ & $25 \%$ & $\mathbf{2 6 \%}$ \\
\hline
\end{tabular}

Fonte: Cálculos elaborados pelos autores com base em Ministério da Agricultura (1995), Madrp (2001) e Autoridade de gestão do Pic LEADER+ (2008).

crescente, apesar de uma média pouco representativa, inferior a $7 \%$ do investimento total realizado pelo setor privado (ver Quadro 4).

Os investimentos do setor público centraram-se, no período em análise, essencialmente na promoção dos recursos endógenos do território e na conservação e preservação do património, por via de investimentos em atividades de marketing e comunicação e de investimentos materiais, como é o caso das obras de recuperação (ver Quadro 5).
No terceiro setor, os investimentos materiais realizados representam, em média, mais de $50 \%$ do montante total despendido. A criação e o desenvolvimento do associativismo de cariz social, cultural e desportivo, foram as atividades mais representadas, juntamente com a promoção dos recursos endógenos, a conservação, dinamização e preservação do património cultural, material e imaterial (ver Quadro 6).

Quadro 4. Investimento realizado pelo setor privado por categoria de investimento e tipologia de atividade/objeto do pedido de apoio, LEADER I - LEADER +, região Alentejo

\begin{tabular}{|c|c|c|c|c|c|}
\hline & & LEADER I & LEADER II & LEADER + & Conjunto \\
\hline \multirow{4}{*}{ Investimento } & Materiais & $97 \%$ & $96 \%$ & $96 \%$ & $96 \%$ \\
\hline & Aquisição de conhecimentos & $1 \%$ & $1 \%$ & $1 \%$ & $1 \%$ \\
\hline & Marketing e comunicação & $2 \%$ & $2 \%$ & $2 \%$ & $2 \%$ \\
\hline & Outras despesas & $0 \%$ & $1 \%$ & $1 \%$ & $1 \%$ \\
\hline \multirow{6}{*}{ Atividade } & Turismo & $61 \%$ & $39 \%$ & $35 \%$ & $44 \%$ \\
\hline & Agroindústria & $19 \%$ & $23 \%$ & $30 \%$ & $25 \%$ \\
\hline & Gastronomia & $5 \%$ & $10 \%$ & $9 \%$ & $8 \%$ \\
\hline & Serviços & $2 \%$ & $10 \%$ & $9 \%$ & $7 \%$ \\
\hline & Comércio & $2 \%$ & $2 \%$ & $8 \%$ & $4 \%$ \\
\hline & Outras atividades & $11 \%$ & $16 \%$ & $9 \%$ & $12 \%$ \\
\hline
\end{tabular}

Fonte: Cálculos elaborados pelos autores com base em Ministério da Agricultura (1995), Madrp (2001) e Autoridade de gestão do Pic LEADER+ (2008).

Quadro 5. Investimento realizado pelo sector público por categoria de investimento e tipologia de atividade/objeto do pedido de apoio, LEADER I - LEADER +, região Alentejo

\begin{tabular}{|c|c|c|c|c|c|}
\hline & & LEADER I & LEADER II & LEADER + & Conjunto \\
\hline \multirow{4}{*}{ Investimento } & Aquisição de conhecimentos & $0 \%$ & $11 \%$ & $7 \%$ & $8 \%$ \\
\hline & Materiais & $64 \%$ & $67 \%$ & $54 \%$ & $60 \%$ \\
\hline & Marketing e comunicação & $36 \%$ & $17 \%$ & $32 \%$ & $27 \%$ \\
\hline & Outras despesas & $0 \%$ & $5 \%$ & $7 \%$ & $6 \%$ \\
\hline \multirow{4}{*}{ Atividade } & Conservação património & $48 \%$ & $45 \%$ & $24 \%$ & $33 \%$ \\
\hline & Promoção do território & $49 \%$ & $21 \%$ & $32 \%$ & $29 \%$ \\
\hline & Formação & $0 \%$ & $15 \%$ & $12 \%$ & $12 \%$ \\
\hline & Outras atividades & $4 \%$ & $19 \%$ & $32 \%$ & $25 \%$ \\
\hline
\end{tabular}

Fonte: Cálculos elaborados pelos autores com base em Ministério da Agricultura (1995), Madrp (2001) 3 Autoridade de gestão do Pic LEADER+ (2008). 
Quadro 6. Investimento realizado pelo $3^{\mathrm{o}}$ sector por categoria de investimento e por tipologia de atividade/objeto do pedido de apoio, LEADER I - LEADER +, região Alentejo

\begin{tabular}{|c|c|c|c|c|c|}
\hline & & LEADER I & LEADER II & LEADER + & Conjunto \\
\hline \multirow{4}{*}{ Investimento } & Aquisição de conhecimentos & $24 \%$ & $23 \%$ & $17 \%$ & $19 \%$ \\
\hline & Materiais & $57 \%$ & $38 \%$ & $60 \%$ & $53 \%$ \\
\hline & Marketing e comunicação & $18 \%$ & $17 \%$ & $10 \%$ & $13 \%$ \\
\hline & Outras despesas & $0 \%$ & $22 \%$ & $13 \%$ & $14 \%$ \\
\hline \multirow{5}{*}{ Atividade } & Conservação património & $14 \%$ & $16 \%$ & $13 \%$ & $14 \%$ \\
\hline & Promoção do território & $13 \%$ & $14 \%$ & $7 \%$ & $10 \%$ \\
\hline & Formação & $5 \%$ & $12 \%$ & $9 \%$ & $10 \%$ \\
\hline & Outras atividades & $68 \%$ & $58 \%$ & $70 \%$ & $66 \%$ \\
\hline & Associativismo & $33 \%$ & $27 \%$ & $42 \%$ & $36 \%$ \\
\hline
\end{tabular}

Fonte: Cálculos elaborados pelos autores com base em Ministério da Agricultura (1995), Madrp (2001) e Autoridade de gestão do Pic LEADER+ (2008).

Os GAL foram as entidades que mais privilegiaram os investimentos imateriais, como é o caso da aquisição e transferência de conhecimentos, nomeadamente através das despesas associadas à contratação de quadros técnicos, à consultadoria externa e à realização de seminários para troca de experiências. No conjunto, mais de $60 \%$ do investimento realizado pelos GAL destinou-se ao desenvolvimento de atividades próprias ao funcionamento destas entidades, enquanto agentes responsáveis pela política de desenvolvimento local. A promoção dos recursos endógenos do território e a qualificação dos recursos humanos foram também atividades com grande expressão no total do investimento realizado, representando $18 \%$ e $13 \%$ do investimento total realizado, respetivamente (ver Quadro 7).

O investimento realizado nos concelhos com uma densidade populacional inferior a $15 \mathrm{hab} / \mathrm{km}^{2}$ apresenta uma tendência decrescente ao longo das três iniciativas LEADER, apesar de, no conjunto, representar cerca de 50\% do montante de investimento realizado. Os concelhos com uma densidade populacional superior a $30 \mathrm{hab} / \mathrm{km}^{2}$, mas inferior a $70 \mathrm{hab} / \mathrm{km}^{2}$, registaram um acréscimo de volume do investimento realizado nestes municípios, atingindo $20 \%$ da despesa total no âmbito do LEADER+. No LEADER I e LEADER II as regiões com um índice de envelhecimento inferior a 150 beneficiaram de $79 \%$ e $53 \%$ do investimento realizado, respetivamente. Neste sentido, pode depreender-se que, dentro das zonas de intervenção dos GAL, as regiões menos envelhecidas foram as principais responsáveis pelo dinamismo dos setores de atividades. No LEADER + assiste-se a uma alteração significativa desta tendência, ou seja, os concelhos com um índice de envelhecimento superior a 150 centralizaram mais de $90 \%$

Quadro 7. Investimento realizado pelos GAL por categoria de investimento por tipologia de atividade/objeto do pedido de apoio, no âmbito do LEADER I - LEADER +, região Alentejo

\begin{tabular}{|l|l|c|c|c|c|}
\hline & & LEADER I & LEADER II & LEADER + & Conjunto \\
\hline \multirow{5}{*}{ Investimento } & Aquisição de conhecimentos & $36 \%$ & $54 \%$ & $53 \%$ & $\mathbf{5 0 \%}$ \\
\cline { 2 - 6 } & Materiais & $12 \%$ & $5 \%$ & $15 \%$ & $\mathbf{1 1 \%}$ \\
\cline { 2 - 6 } & Marketing e comunicação & $33 \%$ & $14 \%$ & $15 \%$ & $\mathbf{1 8 \%}$ \\
\cline { 2 - 6 } & Outras despesas & $18 \%$ & $26 \%$ & $\mathbf{2 1 \%}$ & $\mathbf{6 2 \%}$ \\
\hline \multirow{5}{*}{ Atividade } & Serviços & $38 \%$ & $71 \%$ & $13 \%$ & $\mathbf{1 3 \%}$ \\
\cline { 2 - 6 } & Formação & $15 \%$ & $14 \%$ & $14 \%$ & $\mathbf{1 8 \%}$ \\
\cline { 2 - 6 } & Promoção do território & $35 \%$ & $13 \%$ & $8 \%$ & $\mathbf{7 \%}$ \\
\cline { 2 - 6 } & Outras atividades & $12 \%$ & $2 \%$ & & \\
\hline
\end{tabular}

Fonte: Fonte: Cálculos elaborados pelos autores com base em Ministério da Agricultura (1995), Madrp (2001) e autoridade de gestão do PIC LEADER+ (2008). 
Quadro 8. Investimento realizado por localização geográfico, no âmbito do PIC LEADER I,

LEADER II, LEADER +, na região Alentejo

\begin{tabular}{|c|c|c|c|c|}
\hline & LEADER I & LEADER II & LEADER + & Conjunto \\
\hline \multicolumn{5}{|c|}{ Escalões Densidade Populacional } \\
\hline$<15 \mathrm{hab} / \mathrm{km}^{2}$ & $75 \%$ & $41 \%$ & $43 \%$ & $50 \%$ \\
\hline $15-30 \mathrm{hab} / \mathrm{km}^{2}$ & $25 \%$ & $44 \%$ & $36 \%$ & $36 \%$ \\
\hline$>30 \mathrm{hab} / \mathrm{km}^{2}$ & $0 \%$ & $15 \%$ & $20 \%$ & $14 \%$ \\
\hline \multicolumn{5}{|c|}{ Escalóes Índice de Envelhecimento } \\
\hline$<150$ & $79 \%$ & $53 \%$ & $9 \%$ & $38 \%$ \\
\hline $150-200$ & $17 \%$ & $27 \%$ & $49 \%$ & $35 \%$ \\
\hline$>200$ & $5 \%$ & $20 \%$ & $41 \%$ & $27 \%$ \\
\hline \multicolumn{5}{|c|}{ Escalões Concentração Empresarial na Zona de Intervenção } \\
\hline$<15 \%$ & $20 \%$ & $67 \%$ & $57 \%$ & $50 \%$ \\
\hline $15 \%-30 \%$ & $75 \%$ & $20 \%$ & $23 \%$ & $35 \%$ \\
\hline$>30 \%$ & $5 \%$ & $13 \%$ & $21 \%$ & $15 \%$ \\
\hline
\end{tabular}

Fonte: Cálculos elaborados pelos autores com base em Ministério da Agricultura (1995), Madrp (2001) e Autoridade de gestão do PIC LEADER+ (2008).

do montante de investimento realizado. À exceção dos resultados obtidos no LEADER I, os territórios com uma reduzida concentração empresarial na zona de intervenção do GAL canalizaram mais de $55 \%$ do investimento realizado, fato que nos permite concluir que o programa poderá ter contribuído para fomentar o empreendedorismo em áreas mais carenciadas (ver Quadro 8).

Face à análise descritiva das características do investimento realizado, e comparando os resultados obtidos com os objetivos inicialmente definidos para o Programa LEADER, parece evidente a eficácia deste no estímulo à diversificação de atividades económicas, nas zonas rurais do Alentejo. O LEADER levou, inclusivamente, à especialização do investimento privado em torno de dois setores de atividade: a agro-indústria e o turismo. Estes sectores apresentam-se, hoje em dia, como fileiras económicas estratégicas para o desenvolvimento da região Alentejo (AUTORIDADE DE GESTÃO DO INALENTEJO, 2008).

O volume de emprego nas empresas com atividade económica no domínio do alojamento, restauração e similares, aumentou, entre 1991 e 2010, de 7.200 para 11.800 postos de trabalho, na região Alentejo (INE, 1993 e 2012). Contudo, apesar da iniciativa LEADER ter contribuído para fomentar o empreendedorismo em zonas de menor concentração empresarial (conforme ilustra o Quadro 8), o Programa evidenciou algumas dificuldades em desenvolver uma cultura empresarial ancorada em estratégias inovadoras ${ }^{11}$. Segundo o PORA (AUTORIDADE DE GESTÃO DO INALENTEJO, 2008, p. 42), é ainda persistente hoje em dia no Alentejo, uma cultura empresarial deficitária no que respeita à capacidade de gestão e de implementação de estratégias de marketing e de comercialização.

Por último, importa referir que, apesar dos resultados alcançados pelo Programa ao nível da empregabilidade, a população residente na região Alentejo tem continuado a registar um decréscimo nas últimas décadas, ainda que, a partir de 1991, se tenha verificado uma desaceleração na diminuição da população (SANTOS, 2012, p. 61). Neste contexto, podemos concluir que o LEADER mostrou alguma eficácia no abrandamento do ritmo do êxodo rural, ao ter contribuído para fixar alguma população nas zonas rurais. Paralelamente, o Programa ganhou em eficácia entre o LEADER II e o LEADER +, ao ter conseguido aumentar o volume do investimento nas zonas menos povoadas e mais envelhecidas (ver Quadro 8).

\footnotetext{
11. A análise individual realizada a cada projeto permitiu evidenciar a fraca e/ou ausência de inovação nos projetos financiados, como aliás outros estudos já o haviam demonstrado (TCE, 2010; ÖIR, 2004). Outra evidência sobre o fraco nível de inovação recai no fato de as despesas realizadas em I\&DT serem residuais.
} 


\section{Conclusão}

A análise realizada permitiu concluir que o LEADER, conforme já defendido por Wade e Rinne (2008, p. 82), é efetivamente um Programa com efeitos de longo prazo, ou seja, a sua eficiência tende a aumentar ao longo dos anos ${ }^{12}$. Por outro lado, o estudo evidenciou que o Programa induziu uma especialização do investimento, realizado pelo setor privado, em torno da agro-indústria e do turismo (áreas identificadas nas ELD como de intervenção prioritária).

Marques e Santos (2011) já tinham inclusivamente demonstrado a capacidade das políticas públicas descentralizadas para estimularem trajetórias económicas especializadas. No caso da região Alentejo, as características do território, aliadas à tendência recente de mercado em torno da valorização dos produtos agroalimentares, das produções locais e do turismo, foram propícias ao desenvolvimento dos setores agroindustrial e turístico. A agro-indústria surge como uma solução para aproveitar os recursos primários numa região com forte tradição agrícola (SANTOS, 2012). Ao nível turístico, a região é detentora de um rico património natural e arquitetónico e possui uma forte identidade cultural (AUTORIDADE DE GESTÃO DO INALENTEJO, 2008, p. 45), condição sine qua non para o desenvolvimento destas atividades. No entanto, apesar dos estímulos ao investimento, o Programa mostrou-se pouco eficaz no fomento de atividades de preservação do ambiente ou de projetos inovadores com perfil diferenciador. Por exemplo, os projetos promovidos pelo setor privado tiveram, na sua maioria, como finalidade capacitarem as empresas para o desenvolvimento de uma atividade, em vez de lhes permitirem a obtenção de vantagens competitivas no mercado global.

A análise individual realizada a todos os projetos concretizados, permitiu verificar que a inovação subjacente à maioria das candidaturas não

12. No que respeita ao efeito de alavanca financeira na despesa privada, no cofinanciamento público nacional e no acréscimo do nível de investimento. pode ser vista como radical mas sobretudo de natureza incremental. Não obstante, veio contribuir para a diversificação do sector empresarial e para o aparecimento de novas atividades, numa região onde estas eram escassas e por vezes inexistentes (SANTOS, 2012, p. 63).

Importa ainda referir que todos os beneficiários, à exceção dos GAL, privilegiaram os investimentos de tipo material. As despesas em I\&DT revelaram-se pouco expressivas e/ou totalmente inexistentes, em parte por não terem sido consideradas diretamente como prioritárias nas ELD e por possuírem uma elegibilidade limitada.

Com o apoio da iniciativa LEADER, o Alentejo viu algumas das suas fragilidades serem eliminadas, sobretudo no que respeita à deficiente cobertura de infraestruturas de apoio às atividades produtivas e de alojamento turístico. No entanto, persistem ainda algumas debilidades quanto a: i) iniciativa e densidade empresarial; ii) falta de domínio da cadeia de valor ${ }^{13}$ ou iii) nível de investimento e despesa em I\&DT (AUTORIDADE DE GESTÃO DO INALENTEJO, 2008, p. 42), que importa no futuro ultrapassar.

A melhoria dos resultados (outputs) e dos impactos (outcomes), alcançados por este tipo de políticas públicas, poderia ter sido mais consequente, se estes programas tivessem sido mais orientados para os resultados do que para as realizações. Por exemplo, entre outras alternativas possíveis, mediante a atribuição de um incentivo não reembolsável apenas perante indicadores de desempenho e mérito real. Conforme Bernini e Pellegrini (2011) "as empresas subsidiadas investiram mais do que normalmente o fariam e aumentaram o número de emprego mais do que as empresas não subsidiadas [...]. [Contudo], a produtividade das empresas subsidiadas mostra um menor crescimento do que nas empresas não subsidiadas. As empresas excedem o nível ótimo de

13. "A maioria das empresas não domina a cadeia de valor do seu sector/cluster, carecendo de maior conhecimento nas áreas da organização e gestão, inovação, marketing/ vendas, tecnologias de informação e comunicação, design, investigação e desenvolvimento" (AUTORIDADE DE GESTÃO DO INALENTEJO, 2008, p. 42). 
emprego afim de obterem o subsídio, o que pode afetar a eficiência e crescimento de longo prazo" (BERNINI e PELLEGRINI, 2011, p. 262-264).

\section{Referências bibliográficas}

ARNAUD, S. e BOUDEVILLE, N. Evaluer des politiques et programmes publics. France: Éditions de la Performance, 2004.

AUTORIDADE DE GESTÃO DO INALENTEJO. PORA - Programa Operacional Regional do Alentejo 2007 - 2013. Lisboa: Observatório do QREN - Quadro de Referência Estratégica Nacional, 2008. 152 p.

AUTORIDADE DE GESTÃO DO PIC LEADER+. Listagem dos projectos aprovados. Lisboa: 2008.

BARCA, F., MCCANN, P. e RODRIGUEZ-POSE, A. The Case for Regional Development Intervention: PlacedBased Versus Place Neutral Approaches. Journal of Regional Science, v. 52, n. 1, p. 134-152, 2012.

BARROCAS, C. 15 Anos do Programa LEADER no Alentejo: avaliação de impactos. Beja: Ideia Alentejo - Associação para a Inovação e Desenvolvimento Integrado do Alentejo, 2008.

BARTHELEMY, P. A. e VIDAL, C. Les ruralités de l'Union Européenne. European Comission, 1995. Disponível em: $<$ http://ec.europa.eu/agriculture/envir/report/fr/rur_ fr/report_fr.htm > . Acesso em: 25 mar. 2012.

BERNINI, C. e PELLEGRINI, G. How are growth and productivity in private firms affected by public subsidy? Evidence from a regional policy. Regional Science and Urban Economics, Elsevier B.V, v. 41, Issue 3, p. 253-265, maio 2011.

BERTHET, T. Les enjeux de l'évaluation territoriale des politiques publiques. Informations sociales, v. 2008/6, n. 150, p. 130-139, 2008.

CCRA. Programa de Desenvolvimento Regional: documento de trabalho. Évora: Ministério do Plano e da Administração do Território, 1986.

CE. Valeur Ajoutée Communautaire dans le cadre des Politiques Structurelles - Définition et critères d'évaluation: Document de travail. Bruxelles: Direction Générale, Politique Régionale, 2002. 21 p.

CHAMPETIER, Y. L'Europe et le développement rural. Projet, v. 2003/2, n. 274, p. 59-67, 2003.

CE. L'approche Leader - Guide de base. Luxembourg: Office des publications officielles des Communautés européennes, 2006. 31 p.
CES. Parecer CE (91/C 31/14). Jornal Oficial das Comunidades Europeias, Bruxelas, n. C 31, p. 42-43, 6 fev. 1991.

CESE. Avis du Comité économique et social européen sur le thème: «Leader en tant qu'instrument du développement local» (avis d'initiative), (2011/C 376/03), Journal officiel de l'Union européenne, Bruxelles, n. C 376, p. 15-18, 22 dez. 2011.

DALL'HERBA, S. et al. Fonds structurels, effets de débordement géographique et croissance régionale en Europe. Revue de l'OFCE, v. 2008/1, n. 104, p. 241-269, 2008.

EC. Evalsed, the resource for the Evaluation of SocioEconomic Development - Guide. Luxembourg: Directorate-General for Regional Policy, 2008. 180 p.

Euréval, Centre Européen d'Expertise et d'Evalution. L'Analyse coût-efficacité: fiche téchnique. Eureval. 2 p. Disponível em: <http://www.eureval.fr/IMG/File/FT_ ACE.pdf >. Acesso em: 25 jul. 2013.

FORAY, D. et al. Guide to Research and Innovation Strategies for Smart Specialisation (RIS 3). European Union: Regional Policy, 2012. 122 p. Disponível em: $<$ http://s3platform.jrc.ec.europa.eu/s3pguide>. Acesso em: 5 ago. 2013.

INE. Painel de Empresas 1990-1991. Lisboa: INE, 1993.

INE. Anuário Estatístico da Região Alentejo 2011. Lisboa: INE, 2012.

LAZAREV, G. Promouvoir le développement des territoires ruraux. MediTERRA 2009, Presses de Sciences Po Annuels, CIHEAM et Plan Bleu, p. 183-210, 2009.

MADRP. Programa de Iniciativa Comunitária LEADER II, Relatório de Execução Final. Lisboa: Comissão Nacional de Gestão, Direcção Geral de Desenvolvimento Rural, 2001.

MARQUES, C. e SANTOS, C. Políticas públicas para pensar no desenvolvimento de um sítio local - um modelo. Revista Portuguesa de Estudos Regionais, n. 25-26, p. 107-120, 2011.

MANDL,U.,DIERX, A. e ILZKOVITZ,F. The effectiveness and efficiency of public spending. Economic Paper, n. 301. Brussels: European Commission, Directorate-General for Economic and Financial Affairs, 2008.

MINISTÉRIO DA AGRICULTURA. Programa de Iniciativa Comunitária LEADER, Listagem de projectos Financiados: Volume C e Volume C1. Lisboa: Comissão Nacional de Gestão, Instituto de Estruturas Agrárias e de Desenvolvimento Rural, 1995. 
MORENO, L. O LEADER em Portugal Continental: contexto e elementos de uma análise geográfica de conteúdos. In: V Colóquio Hispano-Português de Estudos Rurais Futuro dos Territórios Rurais numa Europa Alargada, 2003, Bragança. Actas do Colóquio, 2003.

NetO, P., SANTOS, A. e SERRANO, M. M. Public Policies Supporting Local Based Networks for Entrepreneurship and Innovation - Contributions to the Effectiveness and Added Value Assessment. In: Uddevalla Symposium 2012: Entrepreneurship and Innovation Networks, 2012, Faro. Revised papers presented at the 15th Uddevalla Symposium. Sweden: University West, 2012, p. 627-648.

OECD. The New Rural Paradigm: Policies and Governance. OECD Publishing, 2006.

ÖIR. Methods for and Success of Mainstreaming Leader Innovations and Approach into Rural Development Programmes: Final Report. Viena: Commissioned by European Comission, Austrian Institute for Regional Studies and Spatial Planning, 2004. 126 p.

REIMER, B. e MARKEY, S. Place-based Policy: a Rural Perspective. COMMUNITY RESEARCH CONNEXIONS. 2008. 17 p. Disponível em: <http:// crcresearch.org/files-crcresearch_v2/ReimerMarkey
RuralPlaceBasedPolicySummaryPaper20081107.pdf > . Acesso em: 2 jun. 2013.

SANTOS, A. Análise dos efeitos do Programa de Iniciativa Comunitária LEADER na região Alentejo, entre 1991 e 2006. 2012. 83 f. Dissertação (Mestrado em Economia), Universidade de Évora, Évora, 2012.

SAPRU, R. Public Policy: art and craft of policy analysis. Second Edition. New Delhi: Eastern Economy Edition, PHI Learning Private, Limited, 2011.

TCE. Aplicação da abordagem Leader ao desenvolvimento rural: Relatório Especial no 5/2010. Luxemburgo: Serviço das Publicações da União Europeia, 2010. 108 p.

UNIVERSIDADE DE ÉVORA. Programa Operacional Regional do Alentejo 2007-2013, Avaliação Ex-Ante: Relatório Final. Évora: 2007.

VOLLET, D. e HADJAB, F. Manuel de l'évaluation des politiques publiques. France: Editions Quae, 2008.

WADE, P. e RINNE, P. A LEADER Dissemination Guide Book based on programme experience in Finland, Ireland and the Czech Republic. Final Report of the Transnational LEADER Dissemination Project for the Finnish Rural Policy Committee, 2008. 118 p. Disponível em: <http:// ec.europa.eu/agriculture/rur/leaderplus/pdf/library/ cooperation/tnldp.pdf > . Acesso em: 28 mai. 2013. 
